\title{
BENDING AND FREE VIBRATION ANALYSIS OF FUNCTIONALLY GRADED PLATES USING NEW EIGHT- UNKNOWN SHEAR DEFORMATION THEORY BY FINITE ELEMENT METHOD
}

\author{
Nguyen Van Long ${ }^{1}$, Tran Huu Quoc ${ }^{2, *}, \operatorname{Tran}$ Minh $\mathbf{T u}^{2}$ \\ ${ }^{1}$ Construction Technical College No.1, Trung Van, Tu Liem, Ha Noi \\ ${ }^{2}$ University of Civil Engineering, 55 Giai Phong Road, Hai Ba Trung District, Ha Noi \\ *Email: thquoc@gmail.com
}

Received: 16 September 2015: Accepted for publication: 30 December 2015

\begin{abstract}
In this paper, a new eight-unknown shear deformation theory is developed for bending and free vibration analysis of functionally graded plates by finite element method. The theory based on full twelve-unknown higher order shear deformation theory, simultaneously satisfy zeros transverse stresses at top and bottom surface of FG plates. A four-node rectangular element with sixteen degrees of freedom per node is used. Poisson's ratios, Young's moduli and material densities vary continuously in thickness direction according to the volume fraction of constituents which is modeled as power law functions. Results are verified with available results in the literature. Parametric studies are performed for different power law index, side-tothickness ratios.
\end{abstract}

Keywords: functionally graded plate, finite element method, bending, vibration analysis.

\section{INTRODUCTION}

Since it was invented by Japanese scientists in 1984 [1], functionally graded materials (FGMs) are increasingly and widely used in many fields, such as aerospace, marine, mechanical, and structural engineering due to its advantages compared to classical fiberreinforced laminated composites. The typical FGMs composed of ceramic and metal materials. The ceramic composition offers thermal barrier effects and protects the metal from corrosion and oxidation, and the metallic composition provides FGM toughness and strength.

For dynamic and static analysis of functionally graded plates and shells, many plate theories are developed. A review of shear deformation theories for isotropic and laminated plates was carried out by Ghugal and Shimpi [2] and Khandan et al. [3]. Focus on modeling of functionally graded plates and shells, Thai Huu-Tai and Kim Seung-Eock [4] reviewed various theoretical models to investigate their mechanical behavior. The classical plate theory (CPT) based on Kirchhoff assumptions and ignores the transverse shear deformation effect gives appropriate results for thin plates. First-order shear deformation theory (FSDT) takes into 
account the transverse shear deformation effect and needs a shear correction factor which is difficult to determine due to its dependence on many parameters. To overcome the weaknesses of FSDT, the higher-order shear deformation theories are proposed.

A comprehensive review of the various methods employed to study the static, dynamic and stability behavior of functionally graded plates can be found in work of Swaminathan et al. [5]. The review focuses on comparing the stress, vibration and buckling characteristics of FGM plates using different theories. Based on third order shear deformation theory with five displacement unknowns, Reddy [6] developed analytical and finite element solutions for static and dynamic analysis of functionally graded rectangular plates. El-Abbasi and Meguidin [7] used a new thick shell element to study the thermoelastic behavior of functionally graded plates and shells. They extended the four-nodded seven-parameter shell element to account for the varying elastic and thermal properties, as well as the temperature boundary conditions on both faces of FG plates and shells

Oyekoya et al. [8] developed Mindlin-type element and Reissner-type element for the modelling of functionally graded plate subjected to buckling and free vibration. The Mindlintype element formulation is based on averaging of transverse shear distribution over plate thickness using Lagrangian interpolation. The Reissner-type element formulation is based on parabolic transverse shear distribution over plate thickness using Lagrangian and Hermitian interpolation. Talha and Singh [9] studied free vibration and static behavior of functionally graded plates using higher order shear deformation theory. A continuous isoparametric Lagrangian finite element with 13 degrees of freedom per node is employed for the modeling of functionally graded plates. Thai Huu-Tai and Choi Dong-Ho [10] presented finite element formulation of various four-unknown shear deformation theories for the bending and vibration analyses of functionally graded plates. To describe the primary variables, a four-node quadrilateral finite element is developed using Lagrangian and Hermitian interpolation functions. Three-dimensional graded finite element method based on Rayleigh-Ritz energy formulation has been applied to study the static response of the thick functionally graded plates [11].

In this paper, a new higher order displacement field based on twelve-unknown higher order shear deformation theory is developed to analyze the free vibration and buckling of functionally graded plates. The new eight-unknown higher order shear deformation theory is derived from the satisfaction of vanishing transverse shear stress at the top and bottom surfaces of the plate. The finite element model is developed for bending and free vibration analysis of power-law functionally graded plates. A $\mathrm{C}^{1}$ continuous four-node quadrilateral plate element with sixteen degrees of freedom per node is employed. Lagrangian linear interpolation functions are used to describe the in-plane displacements and the rotation of normals about $x, y$ axes; Hermitian cubic interpolation functions are given for the transverse displacement, rotation about $z$-axis, higherorder term of displacements and their first derivation.

\section{KINEMATICS}

The twelve-unknown higher order displacement field is given as follow [12]:

$$
\begin{aligned}
& u(x, y, z)=u_{0}(x, y)+z \theta_{x}(x, y)+z^{2} u_{0}^{*}(x, y)+z^{3} \theta_{x}^{*}(x, y) ; \\
& v(x, y, z)=v_{0}(x, y)+z \theta_{y}(x, y)+z^{2} v_{0}^{*}(x, y)+z^{3} \theta_{y}^{*}(x, y) ; \\
& w(x, y, z, t)=w_{0}(x, y, t)+z \theta_{z}(x, y, t)+z^{2} w_{0}^{*}(x, y, t)+z^{3} \theta_{z}^{*}(x, y, t) .
\end{aligned}
$$


where $u, v, w$ denote the displacements of a point along the $(x, y, z)$ coordinates. $u_{0}, v_{0}, w_{0}$ are corresponding displacements of a point on the midplane. $\theta_{x}, \theta_{y}$ and $\theta_{z}$ are the rotations of the line segment normal to the midplane about the $y$-axis, $x$-axis and $z$-axis , respectively. The functions $u_{0}^{*}, v_{0}^{*}, w_{0}^{*}, \theta_{x}^{*}, \theta_{y}^{*}$ and $\theta_{z}^{*}$ are the higher order terms in the Taylor series expansion defined in the mid-plane.

For bending plates, the transverse shear stresses $\sigma_{x z}, \sigma_{y z}$ must be vanished at the top and bottom surfaces. These conditions lead to the requirement that the corresponding transverse strains on these surfaces be zero. From $\gamma_{x z}\left(x, y, \pm \frac{h}{2}\right)=\gamma_{y z}\left(x, y, \pm \frac{h}{2}\right)=0$, we obtain:

$$
\begin{aligned}
& u_{0}^{*}=-\frac{1}{2} \theta_{z, x}-\frac{h^{2}}{8} \theta_{z, x}^{*} ; \quad \theta_{x}^{*}=-\frac{4}{3 h^{2}}\left(\theta_{x}+w_{0, x}\right)-\frac{1}{3} w_{0, x}^{*} ; \\
& v_{0}^{*}=-\frac{1}{2} \theta_{z, y}-\frac{h^{2}}{8} \theta_{z, y}^{*} ; \quad \theta_{y}^{*}=-\frac{4}{3 h^{2}}\left(\theta_{y}+w_{0, y}\right)-\frac{1}{3} w_{0, y}^{*} .
\end{aligned}
$$

Thus, the displacement field (1) becomes:

$$
\begin{aligned}
& u=u_{0}+z \theta_{x}-\frac{z^{2}}{2}\left(\theta_{z, x}+c_{1} \theta_{z, x}^{*}\right)-\frac{z^{3}}{3}\left[c_{2}\left(\theta_{x}+w_{0, x}\right)+w_{0, x}^{*}\right] \\
& v=v_{0}+z \theta_{y}-\frac{z^{2}}{2}\left(\theta_{z, y}+c_{1} \theta_{z, y}^{*}\right)-\frac{z^{3}}{3}\left[c_{2}\left(\theta_{y}+w_{0, y}\right)+w_{0, y}^{*}\right] \\
& w=w_{0}+z \theta_{z}+z^{2} w_{0}^{*}+z^{3} \theta_{z}^{*} .
\end{aligned}
$$

with: $c_{1}=\frac{h^{2}}{4} ; c_{2}=\frac{4}{h^{2}}$. or in matrix notation as:

$$
\{u\}=[\bar{H}]\{d\} .
$$

where:

$$
[\bar{H}]=\left[\begin{array}{cccccccccccccccc}
1 & 0 & z-\frac{c_{2} z^{3}}{3} & 0 & 0 & \frac{-c_{2} z^{3}}{3} & 0 & 0 & \frac{-z^{2}}{2} & 0 & 0 & \frac{-z^{3}}{3} & 0 & 0 & \frac{-c_{1} z}{2} & 0 \\
0 & 1 & 0 & z-\frac{c_{2} z^{3}}{3} & 0 & 0 & \frac{-c_{2} z^{3}}{3} & 0 & 0 & \frac{-z^{2}}{2} & 0 & 0 & \frac{-z^{3}}{3} & 0 & 0 & \frac{-c_{1} z}{2} \\
0 & 0 & 0 & 0 & 1 & 0 & 0 & z & 0 & 0 & z^{2} & 0 & 0 & z^{3} & 0 & 0
\end{array}\right]
$$

$\{u\}=\{u, v, w\}^{T}$ displacement vector of any generic point within the plate;

$$
\{d\}=\left\{u_{0}, v_{0}, \theta_{x}, \theta_{y}, w_{0}, w_{0, x}, w_{0, y}, \theta_{z}, \theta_{z, x}, \theta_{z, y}, w_{0}^{*}, w_{0, x}^{*}, w_{0, y}^{*}, \theta_{z}^{*}, \theta_{z, x}^{*}, \theta_{z, y}^{*}\right\}^{T} .
$$

Following strain - displacement relation, the non-zero strains are given as: 


$$
\left\{\begin{array}{c}
\varepsilon_{x} \\
\varepsilon_{y} \\
\varepsilon_{z} \\
\gamma_{x y} \\
\gamma_{x z} \\
\gamma_{y z}
\end{array}\right\}=\left\{\begin{array}{c}
\varepsilon_{x}^{0} \\
\varepsilon_{y}^{0} \\
\varepsilon_{z}^{0} \\
\gamma_{x y}^{0} \\
\gamma_{x z}^{0} \\
\gamma_{y z}^{0}
\end{array}\right\}+z\left\{\begin{array}{c}
\kappa_{x}^{0} \\
\kappa_{y}^{0} \\
\kappa_{z}^{0} \\
\kappa_{x y}^{0} \\
\kappa_{x z}^{0} \\
\kappa_{y z}^{0}
\end{array}\right\}+z^{2}\left\{\begin{array}{c}
\varepsilon_{x}^{*} \\
\varepsilon_{y}^{*} \\
\varepsilon_{z}^{*} \\
\gamma_{x y}^{*} \\
\gamma_{x z}^{*} \\
\gamma_{y z}^{*}
\end{array}\right\}+z^{3}\left\{\begin{array}{c}
\kappa_{x}^{*} \\
\kappa_{y}^{*} \\
0 \\
\kappa_{x y}^{*} \\
\kappa_{x z}^{*} \\
\kappa_{y z}^{*}
\end{array}\right\}
$$

or:

$$
\{\varepsilon\}=\left\{\varepsilon^{0}\right\}+\mathrm{z}\left\{\kappa^{0}\right\}+z^{2}\left\{\varepsilon^{*}\right\}+z^{3}\left\{\kappa^{*}\right\}
$$

where:

$$
\begin{aligned}
& \left\{\varepsilon^{0}\right\}=\left\{\varepsilon_{x}^{0}, \varepsilon_{y}^{0}, \varepsilon_{z}^{0}, \gamma_{x y}^{0}\right\}=\left\{u_{0, x}, v_{0, y}, \theta_{z}, u_{0, y}+v_{0, x}\right\} ; \\
& \left\{\kappa_{x}^{0}, \kappa_{y}^{0}, \kappa_{z}^{0}, \kappa_{x y}^{0}\right\}=\left\{\theta_{x, x}, \theta_{y, y}, 2 w_{0}^{*}, \theta_{x, y}+\theta_{y, x}\right\} ; \\
& \left\{\varepsilon_{x}^{*}, \varepsilon_{y}^{*}, \varepsilon_{z}^{*}, \gamma_{x y}^{*}\right\}=\left\{-\frac{1}{2}\left(\theta_{z, x x}+c_{1} \theta_{z, x x}^{*}\right),-\frac{1}{2}\left(\theta_{z, y y}+c_{1} \theta_{z, y y}^{*}\right), 3 \theta_{z}^{*},-\left(\theta_{z, x y}+c_{1} \theta_{z, x y}^{*}\right)\right\} ; \\
& \left\{\kappa_{x}^{*}, \kappa_{y}^{*}, \kappa_{x y}^{*}\right\}=\left\{-\frac{1}{3}\left(c_{2}\left(\theta_{x, x}+w_{0, x x}\right)+w_{0, x x}^{*}\right),-\frac{1}{3}\left(c_{2}\left(\theta_{y, y}+w_{0, y y}\right)+w_{0, y y}^{*}\right),\right. \\
& \left.-\frac{1}{3}\left(c_{2}\left(\theta_{x, y}+\theta_{y, x}+2 w_{0, x y}\right)+2 w_{0, x y}^{*}\right)\right\} ; \\
& \quad\left\{\gamma_{x z}^{0}, \gamma_{y z}^{0}\right\}=\left\{w_{0, x}+\theta_{x}, w_{0, y}+\theta_{y}\right\} ;\left\{\kappa_{x z}^{0}, \kappa_{y z}^{0}\right\}=\left\{-c_{1} \theta_{z, x}^{*},-c_{1} \theta_{z, y}^{*}\right\} ; \\
& \quad\left\{\gamma_{x z}^{*}, \gamma_{y z}^{*}\right\}=\left\{-c_{2}\left(w_{0, x}+\theta_{x}\right),-c_{2}\left(w_{0, y}+\theta_{y}\right)\right\} ;\left\{\kappa_{x z}^{*}, \kappa_{y z}^{*}\right\}=\left\{\theta_{z, x}^{*}, \theta_{z, y}^{*}\right\} .
\end{aligned}
$$

\section{CONSTITUTIVE EQUATION}

Consider a rectangular FGM plate with the length $a$, width $b$, and thickness $h$. The $x$-, $y$-, and z-coordinates are taken along the length, width, and height of the plate, respectively, as shown in Fig. 1. The material properties of FGM plates are assumed to vary continuously through the thickness of the plate by a power law distribution as [6]:

$$
V(z)=\left(V_{c}-V_{m}\right)\left(\frac{z}{h}+\frac{1}{2}\right)^{p}+V_{m}
$$

where $V(z)$ represents the effective material property such as Young's modulus $E$, mass density $\rho$, and Poisson's ratio $v$; subscripts $m$ and $c$ represent the metallic and ceramic constituents, respectively; and $p$ is the volume fraction exponent. 


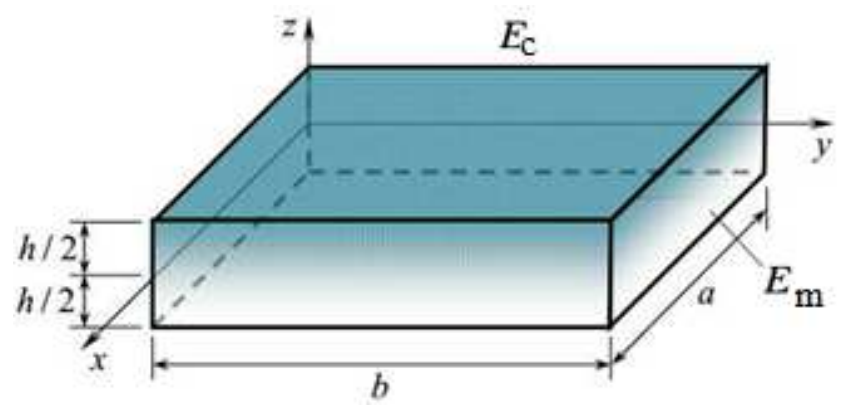

Figure 1. Geometry of FG plate with positive set of reference axes.

The stress-strain relationship for the FGM plate can be written as:

$$
\left\{\begin{array}{c}
\sigma_{x} \\
\sigma_{y} \\
\sigma_{z} \\
\sigma_{x y} \\
\sigma_{x z} \\
\sigma_{y z}
\end{array}\right\}=\left[\begin{array}{cccccc}
Q_{11} & Q_{12} & Q_{13} & 0 & 0 & 0 \\
Q_{21} & Q_{22} & Q_{23} & 0 & 0 & 0 \\
Q_{31} & Q_{32} & Q_{33} & 0 & 0 & 0 \\
0 & 0 & 0 & Q_{44} & 0 & 0 \\
0 & 0 & 0 & 0 & Q_{55} & 0 \\
0 & 0 & 0 & 0 & 0 & Q_{66}
\end{array}\right]\left\{\begin{array}{c}
\varepsilon_{x} \\
\varepsilon_{y} \\
\varepsilon_{z} \\
\gamma_{x y} \\
\gamma_{x z} \\
\gamma_{y z}
\end{array}\right\} \quad \text { or: }\{\sigma\}=[\boldsymbol{D}]\{\boldsymbol{\varepsilon}\}
$$

in which:

$$
\begin{gathered}
Q_{11}=Q_{22}=Q_{33}=\frac{(1-v) E}{(1+v)(1-2 v)} ; Q_{44}=Q_{55}=Q_{66}=\frac{E}{2(1+v)} ; \\
Q_{12}=Q_{23}=Q_{13}=\frac{v E}{(1+v)(1-2 v)}=Q_{21}=Q_{32}=Q_{31} .
\end{gathered}
$$

\section{FINITE ELEMENT FORMULATION}

A $\mathrm{C}^{1}$ continuous four-node quadrilateral plate bending element with sixteen degrees of freedom per node is used (Fig. 2). The Lagrangian linear interpolation functions $N_{i}(\xi, \eta)$ are employed to describe the variables $u_{0}, v_{0}, \theta_{x}, \theta_{y}$ and the Hermitian cubic interpolation functions $H_{i j}(\xi, \eta)$ are employed to describe the variables $w_{0}, w_{0, x}, w_{0, y}, \theta_{z}, \theta_{z, x}, \theta_{z, y}, w_{0}^{*}, w_{0, x}^{*}, w_{0, y}^{*}, \theta_{z}^{*}$ $\theta_{z, x}^{*}, \theta_{z, y}^{*}$ :

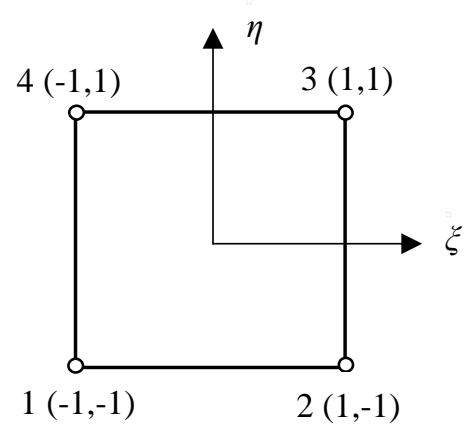

Figure 2. Node number of four-node quadrilateral element in its natural coordinate. 


$$
\begin{gathered}
\left\{u_{0} ; v_{0} ; \theta_{x} ; \theta_{y}\right\}^{T}=\sum_{i=1}^{4} N_{i}\left\{u_{0 i} ; v_{0 i} ; \theta_{x i} ; \theta_{y i}\right\}^{T}=\left[\overline{\boldsymbol{B}}_{\mathbf{1}}\right]\left\{\boldsymbol{q}_{\boldsymbol{e}}\right\} \\
\left\{w_{0} ; \theta_{z} ; w_{0}^{*} ; \theta_{z}^{*}\right\}^{T}=\sum_{i=1}^{4} \sum_{j=1}^{3} H_{i j}\left\{w_{0 i}, w_{0 i, x}, w_{0 i, y} ; \theta_{z i}, \theta_{z i, x}, \theta_{z i, y} ; w_{0 i}^{*}, w_{0 i, x}^{*}, w_{0 i, y}^{*} ; \theta_{z i}^{*}, \theta_{z i, x}^{*}, \theta_{z i, y}^{*}\right\}^{T}=\left[\overline{\boldsymbol{B}}_{21}\right]\left\{\boldsymbol{q}_{e}\right\} \\
\left\{w_{0, x} ; \theta_{z, x} ; w_{0, x}^{*} ; \theta_{z, x}^{*}\right\}^{T}=\sum_{i=1}^{4} \sum_{j=1}^{3} H_{i j, x}\left\{w_{0 i}, w_{0 i, x}, w_{0 i, y} ; \theta_{z i}, \theta_{z i, x}, \theta_{z i, y} ; w_{0 i}^{*}, w_{0 i, x}^{*}, w_{0 i, y}^{*} ; \theta_{z i}^{*}, \theta_{z i, x}^{*}, \theta_{z i, y}^{*}\right\}^{T}=\left[\overline{\boldsymbol{B}}_{22}\right]\left\{\boldsymbol{q}_{e}\right\} \\
\left\{w_{0, y} ; \theta_{z, y} ; w_{0, y}^{*} ; \theta_{z, y}^{*}\right\}^{T}=\sum_{i=1}^{4} \sum_{j=1}^{3} H_{i j, y}\left\{w_{0 i}, w_{0 i, x}, w_{0 i, y} ; \theta_{z i}, \theta_{z i, x}, \theta_{z i, y} ; w_{0 i}^{*}, w_{0 i, x}^{*}, w_{0 i, y}^{*} ; \theta_{z i}^{*}, \theta_{z i, x}^{*}, \theta_{z i, y}^{*}\right\}^{T}=\left[\overline{\boldsymbol{B}}_{23}\right]\left\{\boldsymbol{q}_{e}\right\}
\end{gathered}
$$

For rectangular elements, the interpolation functions $N_{i}$ and $H_{i j}$ for the $i$-th node are given in terms of the natural coordinates as:

$$
\begin{gathered}
N_{i}=\frac{1}{4}\left(1+\xi_{i} \xi\right)\left(1+\eta_{i} \eta\right) \\
H_{i 1}=\frac{1}{8}\left(1+\xi_{i} \xi\right)\left(1+\eta_{i} \eta\right)\left(2+\xi_{i} \xi+\eta_{i} \eta-\xi^{2}-\eta^{2}\right), \\
\mathrm{H}_{i 2}=\frac{1}{8} \xi_{i}\left(\xi_{i} \xi-1\right)\left(1+\eta_{i} \eta\right)\left(1+\xi_{i}^{2} \xi^{2}\right), \\
H_{i 3}=\frac{1}{8} \eta_{i}\left(\eta_{i} \eta-1\right)\left(1+\xi_{i} \xi\right)\left(1+\xi_{i}^{2} \xi^{2}\right) .
\end{gathered}
$$

$\left\{q_{e}\right\}=\left\{q_{1}, q_{2}, q_{3}, q_{4}\right\}^{T}$ is element nodal displacement vector.

$\left\{q_{i}\right\}=\left\{u_{0 i}, v_{0 i}, \theta_{x i}, \theta_{y i}, w_{0 i}, w_{0, x i}, w_{0, y i}, \theta_{z i}, \theta_{z, x i}, \theta_{z, y i}, w_{0 i}^{*}, w_{0, x i}^{*}, w_{0, y i}^{*}, \theta_{z i}^{*}, \theta_{z, x i}^{*}, \theta_{z, y i}^{*}\right\}^{T}$ is nodal displacement vector corresponding to $i$-th node.

The displacement vector at any generic point can be written as:

$$
\{d\}=[\bar{B}]\left\{q_{e}\right\}
$$

where: $[\bar{B}]=\left[\left[\bar{B}_{1}\right],\left[\bar{B}_{21}\right],\left[\bar{B}_{22}\right],\left[\bar{B}_{23}\right]\right]^{T}$ is the shape function matrix.

The strain vector is expressed by:

$$
\{\varepsilon\}=[L]\{d\}=[L][\bar{B}]\left\{q_{e}\right\}=[B]\left\{q_{e}\right\} .
$$

$[L]$ is differential operator matrix, $[B]=[L][\bar{B}]$ is the strain - displacement matrix.

The Hamilton's principle can be expressed as:

$$
0=\int_{0}^{T}(\delta U+\delta W-\delta T) d t
$$

and applying for each element:

The strain energy of the FGM plate element is given by: 


$$
U_{e}=\frac{1}{2} \int_{V_{e}}\{\sigma\}^{T}\{\varepsilon\} d V=\frac{1}{2} \int_{V_{e}}\left\{q_{e}\right\}^{T}[B]^{T}[D][B]\left\{q_{e}\right\} d V=\frac{1}{2}\left\{q_{e}\right\}^{T}\left[K_{e}\right]\left\{q_{e}\right\} .
$$

The external work done on the plate element by distributed applied load may be written as:

$$
W_{e}=-\int_{A_{e}}\{d\}^{T}\{f\} d A=-\int_{A_{e}}\left\{q_{e}\right\}^{T}[\bar{B}]^{T}\{f\} d A=-\left\{q_{e}\right\}^{T}\left\{F_{e}\right\} .
$$

and $\{f\}$ is mechanical load vector.

The kinetic energy of the FGM plate can be expressed as:

$$
T_{e}=\frac{1}{2} \int_{V}\{\dot{u}\}^{T}\{\dot{u}\} \rho(\mathrm{z}) d V=\frac{1}{2} \int_{V}\left\{\dot{q}_{e}\right\}^{T}[\bar{H}]^{T}[B]^{T}[\bar{H}][B]\left\{\dot{q}_{e}\right\} \rho(z) d V=\frac{1}{2}\left\{\dot{q}_{e}\right\}^{T}\left[M_{e}\right]\left\{\dot{q}_{e}\right\} .
$$

Substituting Eqs. (11b-11d) into Eq. (11a), finite element stiffness equation is obtained as:

$$
\left[M_{e}\right]\left\{\ddot{q}_{e}\right\}+\left[K_{e}\right]\left\{q_{e}\right\}=\left\{F_{e}\right\} .
$$

where $\left[K_{e}\right],\left[M_{e}\right]$ and $\left\{F_{e}\right\}$ are the element stiffness matrix, element mass matrix and element nodal load vector, $\left\{q_{e}\right\}$ is nodal displacement vector, and $\left\{\ddot{q}_{e}\right\}$ is the second derivative of the displacements of the element with respect to time.

By assembling the element matrices, the global equilibrium equations for the plate can be obtained as

$$
[K]\{Q\}+[M]\{\ddot{Q}\}=[F] .
$$

where $[K],[M]$ and $\{F\}$ are the global stiffness matrix, mass matrix and nodal load vector of the structure, $\{Q\}$ is nodal displacement vector, and $\{\ddot{Q}\}$ is the second derivative of the displacements of the structures with respect to time.

The generalized governing equation (26) can be employed to study the free vibration and static analysis by dropping the appropriate terms as:

For linear static analysis:

$$
[K]\{Q\}=\{F\} .
$$

For free vibration analysis, the frequency of natural vibration can be obtained from the bellow eigenvalue problem:

$$
\left([K]-\omega^{2}[M]\right)\{Q\}=[0] .
$$

This equation can be solved after imposing boundary conditions of the structure, with eigenvalues solving common problems.

The boundary conditions for an arbitrary edge with simply supported and clamped edge conditions are:

Clamped (C):

$$
u_{0}=v_{0}=\theta_{x}=\theta_{y}=w_{0}=w_{0, x}=w_{0, y}=\theta_{z}=\theta_{z, x}=\theta_{z, y}=w_{0}^{*}=w_{0, x}^{*}=w_{0, y}^{*}=\theta_{z}^{*}=\theta_{z, x}^{*}=\theta_{z, y}^{*}
$$

at $x=0$; a and $y=0$; b.

Simply supported (S):

$$
v_{0}=\theta_{y}=w_{0}=w_{0, y}=\theta_{z}=\theta_{z, y}=w_{0}^{*}=w_{0, y}^{*}=\theta_{z}^{*}=\theta_{z, y}^{*} \text { at } x=0 ; \text { a. }
$$




$$
u_{0}=\theta_{x}=w_{0}=w_{0, x}=\theta_{z}=\theta_{z, x}=w_{0}^{*}=w_{0, x}^{*}=\theta_{z}^{*}=\theta_{z, x}^{*} \text { at } y=0 ; \mathrm{b} \text {. }
$$

\section{NUMERICAL RESULTS}

Matlab codes for finite element model have been built for numerical investigation. After checking convergence, a $10 \times 10$ mesh of four-node element has been used in the computation. The selective integration scheme based on Gauss-quadrature rules, with $3 \times 3$ for membrane, coupling, flexure and inertia terms and $2 \times 2$ for shear term. A rectangular FG plates with different boundary conditions as shown in Fig. 3 are considered (F-free, S-simply supported, and Cclamped). Material properties of the P-FG plate are given in Table 1. For convenience, the following dimensionless forms are used [13]:

$$
\bar{w}=\frac{10 w E_{c} h^{3}}{q_{0} a^{4}} ; \bar{\omega}=\omega h \sqrt{\frac{\rho_{c}}{E_{c}}} .
$$

Table 1. Material properties used in the P-FG plate [13].

\begin{tabular}{llccc}
\hline Properties & $\mathrm{E}(\mathrm{GPa})$ & $v$ & $\rho\left(\mathrm{kg} / \mathrm{m}^{3}\right)$ \\
\hline Metal & Aluminum $(\mathrm{Al})$ & 70 & 0.3 & 2702 \\
Ceramic & Alumina $\left(\mathrm{Al}_{2} \mathrm{O}_{3}\right)$ & 380 & 0.3 & 3800 \\
\hline
\end{tabular}

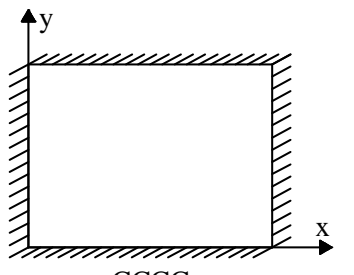

$\mathrm{CCCC}$

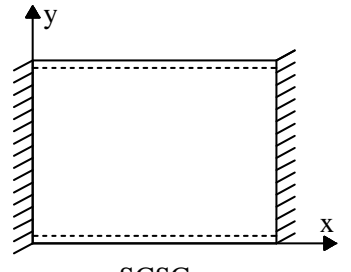

SCSC

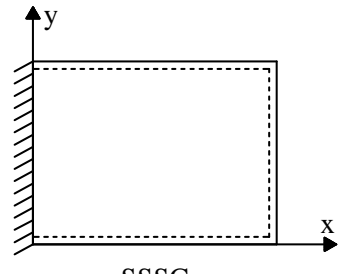

SSSC

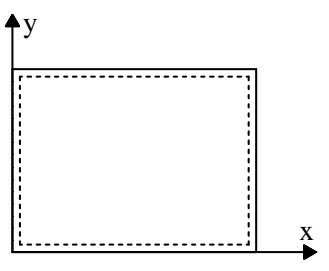

SSSS

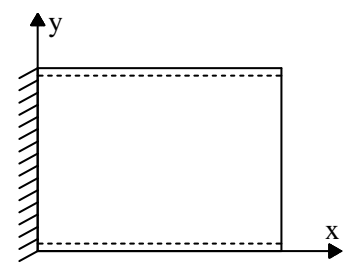

SFSC

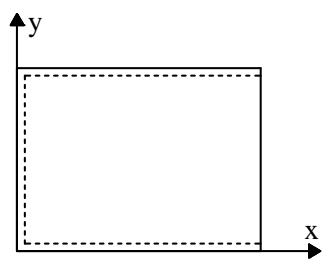

SFSS

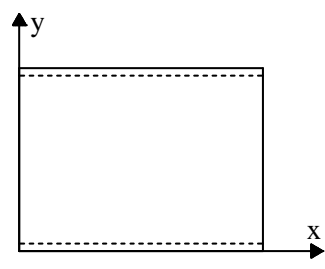

SFSF

Figure 3. Boundary conditions of plates.

Example 1. Validation study

Dimensionless central deflections $\bar{w}$ of isotropic square plates $(p=0)$ with various values of thickness ratios $a / h$ are presented in Table 2. The present results are compared with the solutions given by Thai, H.T., \& Choi, D.H. [10] based on four-unknown shear deformation theories (zeros shape function - FSDT) and the analytical solutions reported by Zenkour [14] based on a mixed first-order shear deformation theory (MPT). It can be seen that the present solution is in 
close agreement with those solutions (errors $<0.2 \%$ ).

Dimensionless fundamental frequencies $\bar{\omega}$ of simply supported (SSSS) square FG plates ( $p=$ 0 ) with various values of thickness ratios $a / h$ and power law index $p$ are presented in Table 3 . The comparison of the dimensionless fundamental frequencies of present results shows good agreement with analytical solutions of Thai H. T., \& Kim S. E. [12] based on simple higher-order theory, and finite element results of Thai H. T., \& Choi D.H. [9] based on four unknowns shear deformation theories.

Table 2. Dimensionless deflection $\bar{w}$ of isotropic square plates under uniform loads.

\begin{tabular}{llcccccc}
\hline \multirow{2}{*}{$a / h$} & \multirow{2}{*}{ Method } & SCSC & SSSC & SSSS & SFSC & SFSS & SFSF \\
\cline { 3 - 7 } & & & Boundary condition \\
\hline 5 & MPT [14] & 0.3021 & 0.3827 & 0.4904 & 0.7139 & 0.9072 & 1.4539 \\
& FSDT [13] & 0.2837 & 0.3686 & 0.4929 & 0.6945 & 0.9146 & 1.4794 \\
& Present & $\mathbf{0 . 2 8 3 3}$ & $\mathbf{0 . 3 5 6 5}$ & $\mathbf{0 . 4 5 2 6}$ & $\mathbf{0 . 6 9 5 8}$ & $\mathbf{0 . 8 8 3 7}$ & $\mathbf{1 . 5 7 4 2}$ \\
10 & MPT [14] & 0.2209 & 0.3059 & 0.4273 & 0.6065 & 0.8224 & 1.3459 \\
& FSDT [13] & 0.2220 & 0.3062 & 0.4298 & 0.6121 & 0.8314 & 1.3722 \\
& Present & $\mathbf{0 . 2 5 5 0}$ & $\mathbf{0 . 3 3 3 7}$ & $\mathbf{0 . 4 3 9 0}$ & $\mathbf{0 . 6 6 2 5}$ & $\mathbf{0 . 8 6 2 9}$ & $\mathbf{1 . 5 4 0 6}$ \\
25 & MPT [14] & 0.1965 & 0.2830 & 0.4096 & 0.5737 & 0.7981 & 1.3154 \\
& FSDT [13] & 0.2047 & 0.2887 & 0.4121 & 0.5890 & 0.8080 & 1.3422 \\
& Present & $\mathbf{0 . 2 0 0 5}$ & $\mathbf{0 . 2 8 1 6}$ & $\mathbf{0 . 3 9 6 1}$ & $\mathbf{0 . 5 8 2 2}$ & $\mathbf{0 . 8 0 0 5}$ & $\mathbf{1 . 4 4 8 7}$ \\
10,000 & MPT [14] & 0.1917 & 0.2785 & 0.4062 & 0.5667 & 0.7931 & 1.3094 \\
& FSDT [13] & 0.2014 & 0.2853 & 0.4087 & 0.5847 & 0.8036 & 1.3365 \\
& Present & $\mathbf{0 . 1 9 1 9}$ & $\mathbf{0 . 2 7 3 6}$ & $\mathbf{0 . 3 9 0 5}$ & $\mathbf{0 . 5 6 9 4}$ & $\mathbf{0 . 7 9 1 8}$ & $\mathbf{1 . 4 3 2 4}$ \\
\hline
\end{tabular}

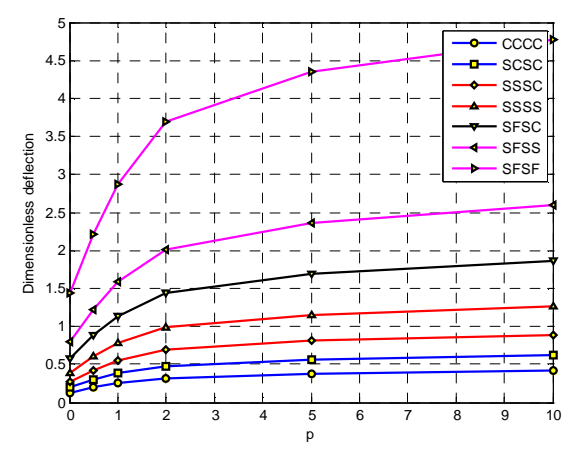

Figure 4. Variation of dimensionless deflection $\bar{w}$ versus power law index $p$ of $\mathrm{Al} / \mathrm{Al} 2 \mathrm{O} 3-1$ square plates under uniform loads $(a / h=10)$.

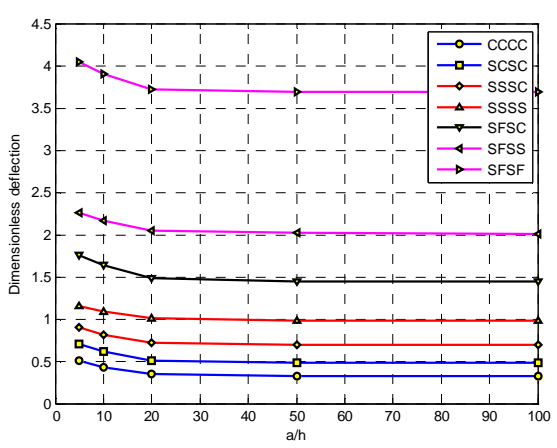

Figure 5. Variation of dimensionless deflection $\bar{w}$ versus thickness ratio $a / h$ of $\mathrm{Al} / \mathrm{Al} 2 \mathrm{O} 3-1$ square plates under uniform loads $(p=2)$. 


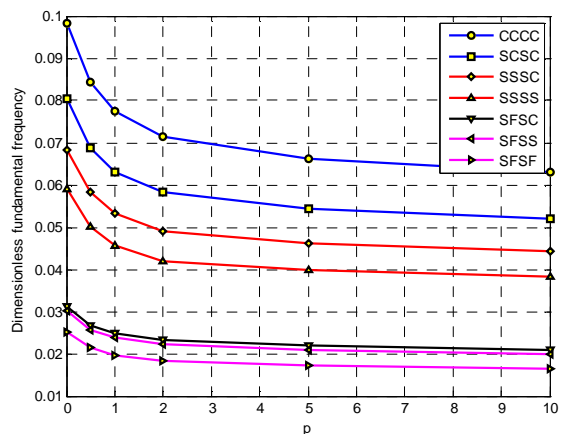

Figure 6. Variation of dimensionless fundamental frequency $\bar{\omega}$ versus power law index $p$ of $\mathrm{Al} / \mathrm{Al}_{2} \mathrm{O}_{3}$ square plates $(a / h=10)$.

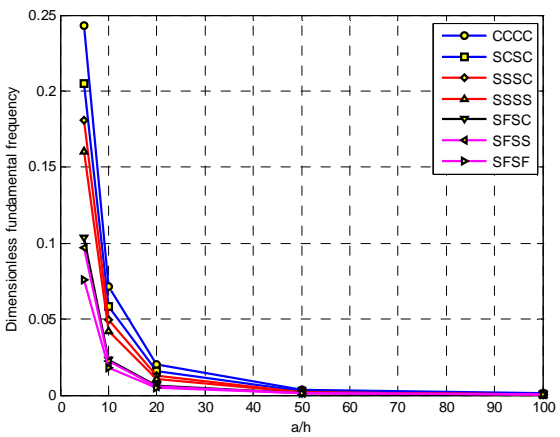

Figure 7.Variation of dimensionless fundamental frequency $\bar{\omega}$ versus thickness ratio a/h of $\mathrm{Al} / \mathrm{Al}_{2} \mathrm{O}_{3}$ square plates $(p=2)$.

Table 3. Dimensionless fundamental frequency $\bar{\omega}$ of $\operatorname{SSSS~Al} / \mathrm{Al}_{2} \mathrm{O}_{3}$ square plates.

\begin{tabular}{llccccc}
\hline \multirow{2}{*}{$a / h$} & \multirow{2}{*}{ Method } & \multicolumn{5}{c}{ Power law index (p) } \\
\cline { 3 - 7 } & TSDT [14] & 0.2113 & 0.1807 & 0.1631 & 0.1378 & 0.1301 \\
& FSDT [13] & 0.2108 & 0.1802 & 0.1629 & 0.1396 & 0.1322 \\
& Present & $\mathbf{0 . 2 2 8 0}$ & $\mathbf{0 . 1 9 4 9}$ & $\mathbf{0 . 1 7 6 5}$ & $\mathbf{0 . 1 5 0 4}$ & $\mathbf{0 . 1 4 2 0}$ \\
\multirow{3}{*}{10} & TSDT [14] & 0.0577 & 0.0490 & 0.0442 & 0.0381 & 0.0364 \\
& FSDT [13] & 0.0576 & 0.0489 & 0.0441 & 0.0382 & 0.0365 \\
& Present & $\mathbf{0 . 0 5 9 1}$ & $\mathbf{0 . 0 5 0 2}$ & $\mathbf{0 . 0 4 5 7}$ & $\mathbf{0 . 0 4 0 2}$ & $\mathbf{0 . 0 3 8 3}$ \\
20 & TSDT [14] & 0.0148 & 0.0125 & 0.0113 & 0.0098 & 0.0094 \\
& Present & $\mathbf{0 . 0 1 5 4}$ & $\mathbf{0 . 0 1 3 0}$ & $\mathbf{0 . 0 1 1 9}$ & $\mathbf{0 . 0 1 0 5}$ & $\mathbf{0 . 0 1 0 0}$ \\
\hline
\end{tabular}

Table 4. Dimensionless deflection $\bar{w}$ of $\mathrm{Al} / \mathrm{Al}_{2} \mathrm{O}_{3}$ square plates under uniform loads.

\begin{tabular}{llllccccc}
\hline \multirow{2}{*}{$a / h$} & $p$ & \multicolumn{7}{c}{ Boundary condition } \\
\cline { 2 - 8 } 5 & 0 & CCCC & SCSC & SSSC & SSSS & SFSC & SFSS & SFSF \\
\hline 5 & 0.2064 & 0.2833 & 0.3565 & 0.4526 & 0.6958 & 0.8837 & 1.5742 \\
& 1 & 0.3048 & 0.4225 & 0.5379 & 0.6909 & 1.0545 & 1.3526 & 2.4082 \\
& 2 & 0.3897 & 0.5418 & 0.6919 & 0.8911 & 1.3602 & 1.7498 & 3.1272 \\
& 5 & 0.5090 & 0.7053 & 0.8956 & 1.1463 & 1.7574 & 2.2511 & 4.0427 \\
& 10 & 0.6757 & 0.9205 & 1.1406 & 1.4234 & 2.2019 & 2.7611 & 4.9461 \\
& 0.7802 & 1.0537 & 1.2921 & 1.5952 & 2.4780 & 3.0770 & 5.5048 \\
10 & 0 & 0.1800 & 0.2550 & 0.3337 & 0.4390 & 0.6625 & 0.8629 & 1.5406 \\
& 0.5 & 0.2720 & 0.3875 & 0.5104 & 0.6756 & 1.0148 & 1.3290 & 2.3678 \\
& 1 & 0.3424 & 0.4899 & 0.6491 & 0.8642 & 1.2974 & 1.7087 & 3.0590 \\
& 2 & 0.4280 & 0.6131 & 0.8144 & 1.0868 & 1.6364 & 2.1622 & 3.9014 \\
& 5 & 0.5271 & 0.7489 & 0.9827 & 1.2960 & 1.9656 & 2.5738 & 4.6574 \\
& 10 & 0.5999 & 0.8469 & 1.1016 & 1.4402 & 2.1933 & 2.8499 & 5.1563 \\
20 & 0 & 0.1393 & 0.2056 & 0.2862 & 0.3996 & 0.5895 & 0.8056 & 1.4562 \\
& 0.5 & 0.2135 & 0.3158 & 0.4411 & 0.6175 & 0.9085 & 1.2445 & 2.2444 \\
& 1 & 0.2725 & 0.4039 & 0.5659 & 0.7945 & 1.1696 & 1.6075 & 2.9111 \\
\hline
\end{tabular}




\begin{tabular}{lllllllll}
\hline & 2 & 0.3429 & 0.5086 & 0.7136 & 1.0029 & 1.4810 & 2.0400 & 3.7224 \\
& 5 & 0.4088 & 0.6042 & 0.8439 & 1.1809 & 1.7518 & 2.4059 & 4.4104 \\
& 10 & 0.4536 & 0.6689 & 0.9309 & 1.2984 & 1.9302 & 2.6432 & 4.8511 \\
& 0 & 0.1297 & 0.1940 & 0.2756 & 0.3919 & 0.5727 & 0.7942 & 1.4395 \\
& 0.5 & 0.2001 & 0.2996 & 0.4259 & 0.6062 & 0.8848 & 1.2280 & 2.2205 \\
1 & 0.2566 & 0.3846 & 0.5478 & 0.7810 & 1.1413 & 1.5877 & 2.8827 \\
& 2 & 0.3231 & 0.4848 & 0.6913 & 0.9863 & 1.4460 & 2.0156 & 3.6873 \\
& 5 & 0.3801 & 0.5698 & 0.8118 & 1.1570 & 1.7013 & 2.3708 & 4.3596 \\
10 & 0.4181 & 0.6263 & 0.8913 & 1.2690 & 1.8679 & 2.6000 & 4.7882 \\
\hline
\end{tabular}

Example 2. Effect of power law index $p$ and side-to-thickness ratio $a / h$ on the dimensionless central deflection $\bar{w}$.

In this example, the square FG plate with different boundary conditions under uniformly distributed load is considered. The calculated dimensionless central deflection with various power law index $p=0 ; 0.5 ; 1.0 ; 2 ; 5 ; 10$ and $a / h=5 ; 10 ; 20 ; 50$ are given in Table 4. Figures 4 and 5 show the variation of power law index $p$ and side-to-thickness ratio $a / h$ versus dimensionless central deflection. It is found that the dimensionless central deflection increases as power law index $p$ increases, while dimensionless central deflection decreases as side-to-thickness ratio increase with all types of boundary conditions.

Table 5. Dimensionless fundamental $\bar{\omega}$ frequency of $\mathrm{Al} / \mathrm{Al}_{2} \mathrm{O}_{3}$ square plates.

\begin{tabular}{lllllllll}
\hline \multirow{2}{*}{$a / h$} & $p$ & \multicolumn{7}{c}{ Boundary condition } \\
\cline { 2 - 8 } & CCCC & SCSC & SSSC & SSSS & SFSC & SFSS & SFSF \\
\hline 5 & 0 & 0.3422 & 0.2896 & 0.2562 & 0.2280 & 0.1480 & 0.1386 & 0.1097 \\
& 0.5 & 0.2970 & 0.2503 & 0.2201 & 0.1949 & 0.1263 & 0.1180 & 0.0933 \\
& 1 & 0.2702 & 0.2274 & 0.1996 & 0.1765 & 0.1143 & 0.1067 & 0.0840 \\
& 2 & 0.2432 & 0.2051 & 0.1806 & 0.1602 & 0.1037 & 0.0968 & 0.0758 \\
& 5 & 0.2174 & 0.1850 & 0.1651 & 0.1482 & 0.0962 & 0.0903 & 0.0706 \\
& 10 & 0.2052 & 0.1755 & 0.1575 & 0.1420 & 0.0924 & 0.0869 & 0.0682 \\
10 & 0 & 0.0984 & 0.0805 & 0.0684 & 0.0591 & 0.0312 & 0.0300 & 0.0252 \\
& 0.5 & 0.0843 & 0.0688 & 0.0582 & 0.0502 & 0.0267 & 0.0256 & 0.0215 \\
& 1 & 0.0775 & 0.0631 & 0.0532 & 0.0457 & 0.0248 & 0.0238 & 0.0197 \\
& 2 & 0.0714 & 0.0582 & 0.0490 & 0.0421 & 0.0233 & 0.0222 & 0.0182 \\
& 5 & 0.0661 & 0.0543 & 0.0461 & 0.0398 & 0.0219 & 0.0209 & 0.0172 \\
& 10 & 0.0630 & 0.0519 & 0.0442 & 0.0383 & 0.0209 & 0.0200 & 0.0165 \\
& 0 & 0.0275 & 0.0220 & 0.0182 & 0.0154 & 0.0080 & 0.0077 & 0.0064 \\
& 0.5 & 0.0234 & 0.0187 & 0.0154 & 0.0130 & 0.0069 & 0.0066 & 0.0055 \\
& 1 & 0.0214 & 0.0171 & 0.0141 & 0.0119 & 0.0064 & 0.0061 & 0.0050 \\
& 2 & 0.0197 & 0.0158 & 0.0130 & 0.0109 & 0.0060 & 0.0057 & 0.0046 \\
& 5 & 0.0187 & 0.0150 & 0.0123 & 0.0104 & 0.0057 & 0.0054 & 0.0044 \\
& 10 & 0.0180 & 0.0144 & 0.0119 & 0.0100 & 0.0054 & 0.0052 & 0.0042 \\
50 & 0 & 0.0046 & 0.0036 & 0.0030 & 0.0025 & 0.0013 & 0.0012 & 0.0010 \\
& 0.5 & 0.0039 & 0.0031 & 0.0025 & 0.0021 & 0.0011 & 0.0011 & 0.0009 \\
\hline
\end{tabular}




\begin{tabular}{llllllll}
\hline 1 & 0.0035 & 0.0028 & 0.0023 & 0.0019 & 0.0010 & 0.0010 & 0.0008 \\
2 & 0.0033 & 0.0026 & 0.0021 & 0.0018 & 0.0010 & 0.0009 & 0.0007 \\
5 & 0.0031 & 0.0025 & 0.0020 & 0.0017 & 0.0009 & 0.0009 & 0.0007 \\
10 & 0.0030 & 0.0024 & 0.0019 & 0.0016 & 0.0009 & 0.0008 & 0.0007 \\
\hline
\end{tabular}

Example 3. Effect of power law index $p$ and side-to-thickness ratio $a / h$ on the fundamental frequency $\bar{\omega}$

Table 5 presents the dimensionless fundamental frequency for various power law index $p=0 ; 0.5$; $1.0 ; 2 ; 5 ; 10$ and $a / h=5 ; 10 ; 20 ; 50$. Different boundary condition for each case is considered. The variation of dimensionless fundamental frequency versus power law index $p$ and side-to-thickness ratio $a / h$ is illustrated in Figures 6 and 7.

It is observed that, for all types of boundary condition, dimensionless frequencies decreases as power law index and side-to-thickness ration increases. Effect of boundary conditions is clearly too, the dimensionless frequency of FG plate with boundary conditions CCCC is highest, and the lowest with SSSS boundary conditions.

\section{CONCLUSIONS}

In this study, the new eight-unknown shear deformation theory is used to analyze the bending and free vibration of rectangular fuctionally graded plates by finite element approach. The governing equations and boundary conditions are derived by employing the Hamilton's principle. Validation studies have been carried out to confirm the accuracy of the present formulation. The obtained result shows a good agreement with those available in the literature. Influence of power law index, side-to-thickness ratio on bending and vibration responses of FG plates have been investigated and discussed. The new eight unknowns shear deformation theory is accurate in predicting static and free vibration responses of FG plates.

\section{REFERENCES}

1. Koizumi M. - FGM activities in Japan, Composites Part B: Engineering 28 (1) (1997) 1-4.

2. Ghugal Y.M, \& Shimpi, R.P. - A review of refined shear deformation theories of isotropic and anisotropic laminated plates, Journal of Reinforced Plastics and Composites 21 (9) (2002) 775-813.

3. Khandan R., Noroozi S., Sewell P., and Vinney J. - The development of laminated composite plate theories: a review, Journal of Materials Science 47 (16) (2012) 59015910.

4. Thai H. T., and Kim S. E. - A review of theories for the modeling and analysis of functionally graded plates and shells, Composite Structures 128 (2015) 70-86.

5. Swaminathan K., Naveenkumar D. T., Zenkour A. M., and Carrera E. - Stress, vibration and buckling analyses of FGM plates - A state-of-the-art review, Composite Structures 120 (2015) 10-31.

6. Reddy J.N. - Analysis of functionally graded plates, International Journal for Numerical Methods in Engineering 47 (2000) 663-684.

7. El-Abbasi N., and Meguid S. A. - Finite element modeling of the thermoelastic behavior of FG plates and shells, Int. J. Comput. Eng. Sci. 1 (2000) 151-165. 
8. Oyekoya O. O., Mba D. U., and El-Zafrany A. M. - Buckling and vibration analysis of functionally graded composite structures using the finite element method, Composite Structures 89 (2009) 134-142.

9. Talha M., and Singh B. N. - Static response and free vibration analysis of FGM plates using higher order shear deformation theory, Applied Mathematical Modelling 34 (2010) 3991-4011.

10. Thai H. T., and Choi D. H. - Finite element formulation of various four unknown shear deformation theories for functionally graded plates, Finite Elements in Analysis and Design 75 (2013) 50-61.

11. Zafarmandand H., and Kadkhodayan M. - Three-dimensional static analysis of thick functionally graded plates using graded finite element method. Proc. I. Mech. E. Part C: J Mechanical Engineering Science 228 (8) (2014) 1275-1285.

12. Jha D. K., Kant T., and Singh R. K. - Higher order shear and normal deformation theory for natural frequency of functionally graded rectangular plates, Nuclear Engineering and Design 250 (2012) 8-13.

13. Thai H. T., and Kim S. E. - A simple higher-order shear deformation theory for bending and free vibration analysis of functionally graded plates, Composite Structures 95 (2013) 188-196.

14. Zenkour A. M. - Exact mixed-classical solutions for the bending analysis of shear deformable rectangular plates, Applied Mathematical Modelling 27 (7) (2003) 515-534.

\title{
TÓM TÁT
}

\section{PHÂN TÍCH UỐN VÀ DAO ĐỌNGTỬ DO CỦA TÂM CÓ CƠ TÍNH BIẾN THIÊN (FGM) BẰNG PHƯƠNG PHÁP PHẦN TƯ HỬU HẠN DỬA TRÊN LÍ THUYÊT TÂMM VỚI 8 ẦN CHUYỂN VI!}

\author{
Nguyễn Văn Long ${ }^{1}$, Trần Hữu Quốc ${ }^{2}$, Trần Minh Tú ${ }^{2}$ \\ ${ }^{1}$ Trường Cao đẳng Xây dưng số 1, Trung Văn, Tù Liêm, Hà Nội \\ ${ }^{2}$ Đại học Xây dụng, 55 Giải Phóng, Quận Hai Bà Trung, Hà Nội \\ *Email:thquoc@gmail.com
}

Bài báo đề xuất lí thuyết tấm biến dạng cắt với 8 thành phần chuyển vị để phân tích uốn và dao động riêng của tấm có cơ tính biến thiên (FGM) bằng phương pháp phần tử hữu hạn. Lí thuyết này được phát triển trên cơ sở lí thuyết tấm bậc ba đầy đủ, đồng thời thoả mãn điều kiện ứng suất ngang tại mặt trên và mặt dưới của tấm bằng không. Mô hình phần tử hữu hạn sử dụng phẩn tử tứ giác 4 nút, mỗi nút 16 bậc tự do. Mô đun đàn hồi kéo (nén), hệ số Poisson và khối lượng riêng của vật liệu biến thiên dọc theo chiều dày tấm theo quy luật hàm mũ. Kết quả tính được so sánh với các kết quả đã công bố của một số tác giả khác cho thấy độ tin cậy của lí thuyết và mô hình tính đã xây dựng. Ảnh hưởng của chỉ số tỉ lệ thể tích, tỉ lệ kích thước hình học... đã được khảo sát.

Tù khoá: tấm FGM, PTHH, dao động tự do, uốn, kết cấu tấm. 